\title{
BMJ Open Physical activity and incident asthma in adults: the HUNT Study, Norway
}

\author{
Ben M Brumpton, ${ }^{1,2,3}$ Arnulf Langhammer, ${ }^{1}$ Manuel A R Ferreira, ${ }^{2}$ Yue Chen, ${ }^{4}$ \\ Xiao-Mei Mai ${ }^{1}$
}

To cite: Brumpton BM,

Langhammer A,

Ferreira MAR, et al. Physical activity and incident asthma in adults: the HUNT Study, Norway. BMJ Open 2016;6: e013856. doi:10.1136/ bmjopen-2016-013856

- Prepublication history and additional material is available. To view please visit the journal (http://dx.doi.org/ 10.1136/bmjopen-2016013856).

Received 11 August 2016 Revised 3 October 2016 Accepted 4 October 2016

For numbered affiliations see end of article.

Correspondence to Dr Ben M Brumpton; ben.brumpton@ntnu.no

\section{ABSTRACT}

Objective: We aimed to investigate the associations of physical activity and change in physical activity with incident asthma in a cohort of Norwegian adults.

Design: We conducted a prospective cohort study using data on self-reported physical activity from 3 waves of the Nord-Trøndelag Health Study. Physical activity was reported at the first and second surveys (1985-1986 and 1995-1997). Physical activity was defined from the second survey as inactive or active and the active group was further defined as very low, low, moderate and high. Change in physical activity was defined from the first and second surveys.

Setting: A large population-based health survey in Norway.

Participants: We followed 18894 adults over 11 years who were free from asthma at baseline in 1995-1997.

Outcome: Incident asthma was reported in the third survey (2006-2008).

Results: The cumulative incidence of asthma was $3.6 \%$ over the 11 years. The adjusted OR for incident asthma among active participants compared with inactive participants was $0.95(95 \% \mathrm{Cl} 0.69$ to 1.34$)$. The adjusted OR for those who were active in the first or second survey versus those who were inactive in both surveys was 0.64 (95\% $\mathrm{Cl} 0.34$ to 1.38); however, this association was strongly attenuated in sensitivity analysis (OR $0.93,95 \% \mathrm{Cl} 0.38$ to 3.09 ).

Conclusions: We did not observe statistically significant associations of physical activity or change in physical activity with incident asthma in adults over the 11-year follow-up.

\section{INTRODUCTION}

Physical activity (PA) has been suggested to be a protective factor against the development of asthma ${ }^{1}$ and, given the strong association between body mass index (BMI) and incident asthma, it could be expected that low levels of PA contribute to this risk. Additionally, studies have found a decrease in the risk of asthma exacerbations among the physically active, ${ }^{2}$ and several cross-sectional studies have found an association between PA and asthma prevalence. ${ }^{3-5}$ However, not all results from cross-sectional studies have been consistent, and longitudinal studies have

\section{Strengths and limitations of this study}

- This study was one of the largest and longest prospective studies on physical activity and incident asthma.

- We had repeated measures of physical activity and comprehensive details of the participants which allowed us to investigate the association in subgroups by age, sex, body mass index, smoking status, family history of asthma and allergic rhinitis.

- Physical activity was self-reported as opposed to objectively measured and the reliance on such self-reported data is a weakness of this study.

- The major criticism of epidemiological studies of asthma and a limitation of our study is the lack of a gold standard for asthma diagnosis; however, previous studies have suggested that the validity and reliability of self-reported asthma are acceptable.

shown conflicting results. ${ }^{6-9}$ A French cohort study which followed 51080 women from 1993 to 2003 found no association between increased PA at baseline and incident asthma (HR 1.00 for the highest tertile of PA compared with lowest $(95 \%$ CI 0.81 to 1.24) ). ${ }^{9}$ On the other hand, in a prospective study of 19021 women from the Australian Longitudinal Study on Women's Health, Lucke et $a l^{10}$ found that middle-aged women (45-50 years) with no or low levels of PA were at a higher risk of incident asthma over a 5-7-year follow-up. This lack of consistency between previous studies should be addressed.

There may exist heterogeneity in effects of PA on asthma, and hidden subgroup effects due to variation in age periods, sex difference and extremes of PA have been suggested. ${ }^{1}$ Additionally, previous studies may suffer from protopathic biases where $\mathrm{PA}$ is restricted due to other respiratory complications that precede asthma or high levels of PA might stimulate respiratory symptoms and advance the detection of asthma. ${ }^{1}$ 
We thus aimed to investigate the associations of PA and change in PA with incident asthma in a relatively large population-based cohort of 18894 adults, over 11 years, as well as investigating the potential associations in subgroups by age, sex, BMI, smoking status, family history of asthma and allergic rhinitis.

\section{METHODS}

\section{Study population}

The Nord-Trøndelag Health Study (HUNT) is a large population-based cohort study that has collected detailed clinical, anthropometric and demographic information over three surveys in the county of Nord-Trøndelag, Norway. The surveys called HUNT1, HUNT2 and HUNT3 were conducted in 1984-1986, 1995-1997 and 2006-2008, respectively. At each survey, the entire adult population (20 years or older) was invited to participate. Participation rates were $89.4 \%$, $69.5 \%$ and $54.1 \%$, respectively. ${ }^{11}$ Detailed information about the HUNT can be found elsewhere. ${ }^{11-13}$ Our analysis included participants who participated in HUNT2 and HUNT3 ( $\mathrm{n}=37071)$ with an average follow-up of 11 years. We excluded those who were $\geq 65$ years of age in HUNT3, did not have information on PA or asthma, leaving 18894 adults in our analysis cohort. The age cutpoint was used to reduce misclassification of chronic obstructive pulmonary disease (COPD) and asthma. Additionally, we conducted analysis on 12707 participants who had information on PA from HUNT1 and HUNT2.

\section{Physical activity}

In HUNT2, participants were asked to record their average hours of light and hard PA during leisure time per week in the past year (none, <1, 1-2, 3 hours or more). Light activity was defined as no sweating or not being out of breath, and hard activity as sweating/out of breath. We defined inactive individuals as those who reported no light or hard PA, and active individuals as those who reported either light or hard PA. We further defined the active individuals into four categories (very low, low, moderate and high). Very low PA was defined as $<3$ hours of light activity and no hard activity, low PA as 3 hours or more of light activity and no hard activity or $<1$ hour of hard activity with $<3$ hours of light activity, and moderate PA as 3 hours or more of light activity and $<1$ hour of hard activity or 1-2 hours hard activity with any light activity. High PA was defined as 3 hours or more of hard activity regardless of light activity. A moderate or high level of PA was assumed to be comparable to the current PA recommendations.

In HUNT1, participants were asked 'How often do you exercise? (never, less than once a week, 2-3 times a week and nearly every day)' with the accompanying text 'By exercise we mean going for walks, skiing, swimming and working out/sports'. We defined inactive individuals as those who answered never and active individuals as those who answered any other category. We then combined the inactive and active categories from HUNT1 and HUNT2 to create four categories: (1) inactive in both; (2) active only in HUNT1; (3) active only in HUNT2 and (4) active in both.

\section{Asthma}

In HUNT2 and HUNT3, participants were asked: 'Do you have or have you had asthma?'. Incident asthma was defined from the survey questionnaires as those who did not report ever asthma at HUNT2 but reported asthma at HUNT3. In a sensitivity analysis, we included a stricter definition of asthma, that is, self-reported incident asthma as above and use of asthma medication at follow-up, without a report of attacks of wheezing at baseline versus the reference group with no asthma and no wheeze at baseline or follow-up. Participants were asked 'Have you at any time during the past 5 years taken medicine for asthma, chronic bronchitis, emphysema or COPD?' at HUNT3 and 'Have you had attacks of wheezing or breathlessness during the past 12 months?' at HUNT2 which we used to define medication use and wheezing, respectively.

\section{Covariates}

In HUNT2, information on health and lifestyle factors was collected in questionnaires and a clinical examination. Information included age, sex, BMI, smoking status (never, former, current, unknown), education $(<10,10-12, \geq 13$, unknown), family history of asthma (yes, no, unknown), social benefit (yes, no, unknown), economic difficulties (yes, no, unknown) and allergic rhinitis (yes, no, unknown).

BMI was calculated as weight (kilograms) divided by the squared value of height (metres). To define smoking status, participants reported whether they were never-smokers, current smokers and age of cessation. The variable was corrected with information from participants' answers in HUNT1 and HUNT3. Family history of asthma included any first-degree family member (father, mother, brother or sister) with asthma. Social benefit included participants who received sick pay, rehabilitation benefits, retraining benefits, disability pension, old age pension, family income supplement, unemployment benefits, transitional benefits, widow's pension or any other benefit. Economic difficulties included participants who had difficulties meeting the cost of food, transport and/or housing. Allergic rhinitis included participants who reported hay fever or nasal allergies.

\section{Statistical methods}

We described the baseline characteristic of the analysis cohort in table 1. Mean and SD or number of observations and percentage for different characteristics of the analysis cohort are presented. The 11-year cumulative incidence of asthma was calculated according to PA. 
Table 1 Baseline characteristics of the analysis cohort, Nord-Trøndelag Health Study, Norway, 1995-1997 ( $n=18$ 894)

\begin{tabular}{|c|c|c|c|c|c|c|c|c|c|c|}
\hline \multirow{2}{*}{ Characteristic } & \multicolumn{2}{|c|}{ Inactive } & \multicolumn{2}{|c|}{ Very low } & \multicolumn{2}{|l|}{ Low } & \multicolumn{2}{|c|}{ Moderate } & \multicolumn{2}{|l|}{ High } \\
\hline & Mean & SD & Mean & SD & Mean & SD & Mean & SD & Mean & SD \\
\hline Age & 39.3 & 8.8 & 40.2 & 8.6 & 39.8 & 8.7 & 39 & 8.8 & 37.6 & 9.7 \\
\hline \multirow[t]{2}{*}{ BMI } & 26.3 & 4.3 & 26 & 4 & 25.8 & 3.8 & 25.4 & 3.5 & 25.2 & 3.3 \\
\hline & $\mathbf{n}$ & Per cent & $\mathbf{n}$ & Per cent & $\mathbf{n}$ & Per cent & $\mathbf{n}$ & Per cent & $\mathbf{n}$ & Per cent \\
\hline \multicolumn{11}{|l|}{ Sex } \\
\hline Men & 543 & 56.2 & 1482 & 37.4 & 2170 & 45.7 & 3103 & 45.8 & 1561 & 64.2 \\
\hline Women & 423 & 43.8 & 2482 & 62.6 & 2583 & 54.3 & 3675 & 54.2 & 872 & 35.8 \\
\hline \multicolumn{11}{|c|}{ Family history of asthma } \\
\hline No & 739 & 76.5 & 3075 & 77.6 & 3710 & 78.1 & 5313 & 78.4 & 1925 & 79.1 \\
\hline Yes & 133 & 13.8 & 583 & 14.7 & 715 & 15.0 & 998 & 14.7 & 328 & 13.5 \\
\hline Don't know & 94 & 9.7 & 306 & 7.7 & 328 & 6.9 & 467 & 6.9 & 180 & 7.4 \\
\hline \multicolumn{11}{|l|}{ Education } \\
\hline Low & 301 & 31.2 & 905 & 22.8 & 836 & 17.6 & 851 & 12.6 & 355 & 14.6 \\
\hline Mid & 546 & 56.5 & 2174 & 54.8 & 2584 & 54.4 & 3553 & 52.4 & 1300 & 53.4 \\
\hline High & 109 & 11.3 & 857 & 21.6 & 1302 & 27.4 & 2344 & 34.6 & 759 & 31.2 \\
\hline Missing & 10 & 1.0 & 28 & 0.7 & 31 & 0.7 & 30 & 0.4 & 19 & 0.8 \\
\hline \multicolumn{11}{|l|}{ Social benefit } \\
\hline No & 547 & 56.6 & 2443 & 61.6 & 3138 & 66.0 & 4560 & 67.3 & 1618 & 66.5 \\
\hline Yes & 219 & 22.7 & 748 & 18.9 & 797 & 16.8 & 1043 & 15.4 & 337 & 13.9 \\
\hline Missing & 200 & 20.7 & 773 & 19.5 & 818 & 17.2 & 1175 & 17.3 & 478 & 19.6 \\
\hline \multicolumn{11}{|c|}{ Economic difficulties } \\
\hline No & 480 & 49.7 & 2226 & 56.2 & 2811 & 59.1 & 4108 & 60.6 & 1484 & 61.0 \\
\hline Yes & 326 & 33.7 & 1140 & 28.8 & 1334 & 28.1 & 1811 & 26.7 & 566 & 23.3 \\
\hline Missing & 160 & 16.6 & 598 & 15.1 & 608 & 12.8 & 859 & 12.7 & 383 & 15.7 \\
\hline \multicolumn{11}{|l|}{ Smoking status } \\
\hline Never & 349 & 36.1 & 1672 & 42.2 & 2188 & 46.0 & 3405 & 50.2 & 3405 & 50.2 \\
\hline Former & 232 & 24.0 & 958 & 24.2 & 1230 & 25.9 & 1674 & 24.7 & 1674 & 24.7 \\
\hline Current & 375 & 38.8 & 1311 & 33.1 & 1312 & 27.6 & 1653 & 24.4 & 1653 & 24.4 \\
\hline Missing & 10 & 1.0 & 23 & 0.6 & 23 & 0.5 & 46 & 0.7 & 46 & 0.7 \\
\hline \multicolumn{11}{|l|}{ Allergic rhinitis } \\
\hline No & 649 & 67.2 & 2678 & 67.6 & 3283 & 69.1 & 4640 & 68.5 & 1604 & 65.9 \\
\hline Yes & 111 & 11.5 & 570 & 14.4 & 711 & 15.0 & 1031 & 15.2 & 362 & 14.9 \\
\hline Missing & 206 & 21.3 & 716 & 18.1 & 759 & 16.0 & 1107 & 16.3 & 467 & 19.2 \\
\hline Total & 966 & 5.1 & 3964 & 21.0 & 4753 & 25.2 & 6778 & 35.9 & 2433 & 12.9 \\
\hline
\end{tabular}

We used logistic regression to investigate the prospective association between levels of $\mathrm{PA}$ and subsequent risk of incident asthma. Each category of PA was compared with the inactive group. We repeated the above analyses using the stricter definition of asthma.

We included the potential confounding factors age, sex, BMI, smoking status, education, family history of asthma, social benefit, economic difficulties and allergic rhinitis in our models. We conducted several stratified analyses to assess whether the association between PA and incident asthma could be modified by other factors. This included stratification by age (dichotomised by age $<45$ years), sex, BMI (dichotomised by $\mathrm{BMI}<25$ ), smoking status (never-smoker, ever-smoker), family history of asthma (yes, no) and allergic rhinitis (yes, no). We formally tested the homogeneity of stratumspecific relative risks using a likelihood ratio test. Finally, we performed several sensitivity analyses. To address potential protopathic biases from other respiratory complications, we excluded participants who reported wheezing, breathlessness or coughing daily during the past 12 months in HUNT2. Additionally, in the analyses of change in PA, we excluded those reporting normally coughing in the morning or normally expectorating phlegm from their chest in the morning in HUNT1.

The statistical analyses were conducted using R V.3.2.2 for Windows ( $\mathrm{R}$ Core Team. R: a language and environment for statistical computing. Vienna, Austria: R Foundation for Statistical Computing, 2012. ISBN 3-900051-07-0 2014).

All participants gave informed written consent.

\section{RESULTS}

Table 1 presents the characteristics of the analysis cohort according to the level of PA. The proportions of inactive, very low, low, moderate and high PA levels in HUNT2 were $5.1 \%, 21.0 \%, 25.2 \%, 35.9 \%$ and $12.9 \%$, respectively. The 11-year cumulative incidence of asthma was $3.6 \%$. The mean age and SD was similar in each category of 
Table 2 The association between PA at baseline and incident asthma in the Nord-Trøndelag Health Study, Norway, 19951997 to $2006-2008(n=18$ 894)

\begin{tabular}{|c|c|c|c|c|c|c|c|c|}
\hline \multirow[b]{2}{*}{ Level of PA } & \multirow[b]{2}{*}{$\mathbf{n}$} & \multirow[b]{2}{*}{ Cases } & \multicolumn{2}{|c|}{ Model 1* } & \multicolumn{2}{|c|}{ Model 2† } & \multicolumn{2}{|c|}{ Model 3‡ } \\
\hline & & & OR & $95 \% \mathrm{Cl}$ & $\overline{\text { OR }}$ & $95 \% \mathrm{Cl}$ & OR & $95 \% \mathrm{Cl}$ \\
\hline Inactive & 966 & 41 & Ref & & Ref & & Ref & \\
\hline Very low & 3964 & 147 & 0.87 & 0.62 to 1.25 & 0.92 & 0.65 to 1.34 & 0.91 & 0.64 to 1.32 \\
\hline Low & 4753 & 170 & 0.84 & 0.60 to 1.20 & 0.96 & 0.68 to 1.38 & 0.95 & 0.67 to 1.37 \\
\hline Moderate & 6778 & 225 & 0.77 & 0.56 to 1.10 & 0.93 & 0.66 to 1.33 & 0.91 & 0.65 to 1.31 \\
\hline High & 2433 & 94 & 0.91 & 0.63 to 1.33 & 1.19 & 0.82 to 1.76 & 1.17 & 0.80 to 1.73 \\
\hline Any activity & 18894 & 677 & 0.83 & 0.61 to 1.16 & 0.97 & 0.71 to 1.36 & 0.95 & 0.69 to 1.34 \\
\hline
\end{tabular}

The 11-year cumulative incidence of asthma was calculated according to PA.

${ }^{*}$ Model 1: crude.

†Model 2: adjusted age, BMI, sex, smoking status, education, family history of asthma, social benefit and economic difficulties.

†Model 3: adjusted age, BMI, sex, smoking status, education, family history of asthma, social benefit, economic difficulties and allergic rhinitis. BMI, body mass index; $n$, number of observations; PA, physical activity.

PA. There were fewer women in the inactive group than in the combined active group. A similar proportion of participants had a family history of asthma between the categories of PA. Participants with higher levels of PA seemed to have higher levels of education, to report less social benefit and economic difficulties and were more likely to be never-smokers compared with inactive people. The proportion of participants who reported allergic rhinitis was slightly lower in the inactive group compared with all physically active groups.

Table 2 presents the crude and adjusted ORs and 95\% CIs for incident asthma in relation to levels of PA. Being active at different levels was not associated with a decreased risk of incident asthma compared with those who reported being inactive. After adjustment for potential confounding factors, the ORs were attenuated and close to null.

The crude OR for incident asthma for persons being active at both surveys compared with inactive at both surveys was 0.58 (95\% CI 0.31 to 1.23 ; table 3 ). After adjustment for potential confounding factors, the OR was 0.66 (95\% CI 0.35 to 1.44$)$. The adjusted OR for any activity in either survey versus inactive at both surveys was 0.64 (95\% CI 0.34 to 1.38 ).
Restricting our analysis to those reporting asthma and use of asthma medication at follow-up, without report of asthma or wheeze at baseline did not change our estimates for the association between PA in HUNT2 and incident asthma (see online supplementary table S1). However, for the association between change in PA prior to baseline and incident asthma, it strongly attenuated the association (any activity vs inactive adjusted OR 0.93 , 95\% CI 0.38 to 3.09, see online supplementary table S2).

The association between PA in HUNT2 and incident asthma did not differ in analyses stratified by age, sex, BMI, smoking status, family history of asthma and allergic rhinitis. The $\mathrm{p}$ values for interaction were $0.95,0.18$, $0.80,0.28,0.40$ and 0.60 , respectively. Regarding the association between change in PA prior to baseline and incident asthma, $\mathrm{p}$ values for interaction were $0.58,0.06$, $0.11,0.12,0.33$ and 0.93 , respectively.

In the analyses excluding participants who reported other respiratory symptoms at HUNT1 (normally coughing in the morning or normally expectorating phlegm from their chest in the morning) or HUNT2 (wheezing, breathlessness or coughing daily during the past 12 months), the estimates did not change considerably (see online supplementary tables S3 and S4).

Table 3 The association between change in PA prior to baseline and incident asthma in the Nord-Trøndelag Health Study, Norway, $1984-1986$ to $2006-2008(n=12707)$

\begin{tabular}{|c|c|c|c|c|c|c|c|c|}
\hline \multirow[b]{2}{*}{ Level of physical activity } & \multirow[b]{2}{*}{$\mathbf{N}$} & \multirow[b]{2}{*}{ Cases } & \multicolumn{2}{|c|}{ Model $1^{*}$} & \multicolumn{2}{|c|}{ Model 2† } & \multicolumn{2}{|c|}{ Model 3‡ } \\
\hline & & & $\overline{\text { OR }}$ & $95 \% \mathrm{Cl}$ & $\overline{\text { OR }}$ & $95 \% \mathrm{Cl}$ & $\overline{\text { OR }}$ & $95 \% \mathrm{Cl}$ \\
\hline Inactive & 150 & 9 & Ref & & Ref & & Ref & \\
\hline Active survey 1 only & 374 & 10 & 0.43 & 0.17 to 1.10 & 0.4 & 0.16 to 1.05 & 0.41 & 0.16 to 1.06 \\
\hline Active survey 2 only & 815 & 25 & 0.50 & 0.23 to 1.14 & 0.53 & 0.25 to 1.23 & 0.53 & 0.24 to 1.23 \\
\hline Active both & 11368 & 404 & 0.58 & 0.31 to 1.23 & 0.68 & 0.36 to 1.45 & 0.66 & 0.35 to 1.44 \\
\hline Any activity & 12557 & 439 & 0.57 & 0.30 to 1.21 & 0.65 & 0.34 to 1.40 & 0.64 & 0.34 to 1.38 \\
\hline
\end{tabular}

The 11-year cumulative incidence of asthma was calculated according to PA.

*Model 1: crude.

†Model 2: adjusted age, BMI, sex, smoking status, education, family history of asthma, social benefit and economic difficulties.

$\ddagger$ Model 3: adjusted age, BMI, sex, smoking status, education, family history of asthma, social benefit, economic difficulties and allergic rhinitis. $\mathrm{BMI}$, body mass index; $\mathrm{n}$, number of observations; PA, physical activity. 


\section{DISCUSSION}

In this prospective study of 18894 adults, we did not observe a decreased risk of incident asthma in physically active compared with inactive adults over the 11-year follow-up. Despite observing a protective point estimate for those physically active at any or both surveys, the estimate had a high degree of uncertainty and was not supported in our sensitivity analyses. We did not find any variation in subgroups by age, sex, BMI, smoking status, family history of asthma or allergic rhinitis. Finally, after restricting to participants without any other respiratory symptoms at baseline, we found similar results, suggesting that the findings are not affected by protopathic biases.

To the best of our knowledge, this was the longest study to investigate the association between PA and incident asthma. The association between PA and asthma has been previously investigated in several cross-sectional and prospective studies. In a systematic review and meta-analysis, Eijkemans and colleagues reported on 34 cross-sectional studies which investigated PA and asthma prevalence. Thirteen of these studies reported an association between higher PA levels and lower asthma prevalence, 18 no association and 3 reported an association between higher PA levels and higher asthma prevalence. ${ }^{1}$ In the same investigation, five longitudinal studies investigating physical actively levels and incident asthma were pooled. The duration of these studies ranged between 5 and 10 years and the studies were largely heterogeneous from each other $\left(\chi^{2}\right.$ test for heterogeneity, $\mathrm{p}=0.07)$. The pooled OR for incident asthma was 0.88 (95\% CI 0.77 to 1.01 ), suggesting PA as a protective factor. This meta-analysis, however, did not control for potential confounding factors as the majority of the individual studies did not provide adjusted results and were unable to investigate possible subgroup variations. Following this meta-analysis, two additional studies have been published. ${ }^{14}{ }^{15}$ The first did not find that PA was associated with wheezing or shortness of breath in children. ${ }^{14}$ The second, which followed 1369 children to adulthood, found a possible beneficial effect of PA on the development of respiratory symptoms (OR 0.97, $95 \%$ CI 0.95 to 0.99$).^{15}$

Despite having a reasonably large sample size, long follow-up and comprehensive details of the participants, there are some potential limitations of our study. First, the definition of asthma was based on self-reported information and it is possible that disease misclassification may have influenced the results. However, it has been reported that the validity of self-reported asthma is reasonably good. ${ }^{16}$ Additionally, we used a stricter definition of asthma based on reported asthma and medication use among those who did not report asthma or wheeze at baseline. This may have improved the validity of the diagnosis and reduced recall bias as the outcome window for medication use was 5 years.

An additional limitation of this study was that PA was subjective and self-reported in questionnaires as opposed to objective measurements such as motion sensors. However, the reliability and validity of the questionnaire used to define PA in HUNT2 have been investigated, and the question on hard PA, which predominantly defined the two highest categories of PA (moderate and high), correlated well with other measures including maximum oxygen uptake $\left(\mathrm{VO}_{2 \max }\right) .{ }^{17}$ The question on PA in HUNT1 has also been validated. ${ }^{18}$ The question on frequency showed high repeatability (Spearman's correlation coefficient 0.87), reliability (weighted $\kappa$ coefficient 0.80 ) and moderate correlation with $\mathrm{VO}_{2 \max }$ (Spearman's correlation coefficient 0.43). ${ }^{18}$ Despite not using an objective measure of $\mathrm{PA}$, which is not very practical in large-scale epidemiological studies, by evaluating PA at two different time points we may have reduced the chance of spurious results due to exposure misclassification.

Observational studies inherently suffer from confounding. Previous studies have hypothesised that protopathic biases where PA is restricted due to other respiratory complications that precede asthma or high levels of PA that stimulate symptoms and advance the detection of asthma may overestimate or underestimate the association, respectively. ${ }^{1}$ To investigate this potential bias, first we excluded those who reported wheezing or coughing daily at HUNT2 $(n=16079)$. We observed a similar OR to our main analysis (adjusted OR for any activity was 0.90 vs 0.95 ; see online supplementary table S3). Second, in the analysis of change in PA, we additionally excluded those who reported normally coughing in the morning or normally expectorating phlegm in the morning at HUNT1. We observed a slightly elevated OR (adjusted OR for any activity in the first or second survey was 0.83 vs 0.64 in the main analysis; see online supplementary table S4), which suggested that our original results for change in PA may have been overestimated due to other respiratory complications. Additionally, we speculated that even if those with a high level of PA were diagnosed earlier than other participants, resulting in an underestimation of the association, we might expect to see an inverse association in those with low or moderate PA compared with inactive participants, but there was no statistically significant association in our findings. It is possible that there is a weak association between PA and incident asthma which can only be detected with very precise measurements of PA and asthma.

Finally, our study was performed in a relatively uniform population in terms of age, ethnicity $(97 \%$ Caucasians) and environmental factors; therefore, our findings may not be directly generalised to other populations.

\section{CONCLUSIONS}

In summary, we did not find strong evidence that higher PA levels or PA overtime are protective against asthma 
development. Future prospective studies with objectively measured PA and larger population size may help to shed light on this association.

\section{Author affiliations}

${ }^{1}$ Faculty of Medicine, Department of Public Health and General Practice, Norwegian University of Science and Technology, NTNU, Trondheim, Norway ${ }^{2}$ QIMR Berghofer Medical Research Institute, Brisbane, Queensland, Australia ${ }^{3}$ Department of Thoracic and Occupational Medicine, St. Olavs Hospital, Trondheim University Hospital, Trondheim, Norway

${ }^{4}$ Faculty of Medicine, School of Epidemiology, Public Health and Preventive Medicine, University of Ottawa, Ottawa, Ottawa, Canada

Acknowledgements The Nord-Trøndelag Health Study (HUNT) is a collaboration between HUNT Research Centre (Faculty of Medicine, Norwegian University of Science and Technology, NTNU), Nord-Trøndelag County Council, Central Norway Health Authority and the Norwegian Institute of Public Health.

Contributors BMB and X-MM conceived and designed the study. AL and $B M B$ acquired the samples and analysed the data. BMB, AL, MARF, YC and $X$-MM interpreted the data. BMB drafted the manuscript. BMB, AL, MARF, YC and $X-M M$ wrote and revised the manuscript. BMB is accountable for the accuracy and integrity of all parts of the work. BMB, AL, MARF, YC and X-MM read and approved the final manuscript to be published.

Funding This work was supported by the Liaison Committee between the Central Norway Regional Health Authority and the Norwegian University of Science and Technology.

Competing interests BMB reported having received funding support from the Central Norway Regional Health Authority and the Norwegian University of Science and Technology.

Ethics approval The Regional Committee for Medical Research Ethics, Norway (2014/102/REK midt).

Provenance and peer review Not commissioned; externally peer reviewed.

Data sharing statement No additional data are available.

Open Access This is an Open Access article distributed in accordance with the Creative Commons Attribution Non Commercial (CC BY-NC 4.0) license, which permits others to distribute, remix, adapt, build upon this work noncommercially, and license their derivative works on different terms, provided the original work is properly cited and the use is non-commercial. See: http:// creativecommons.org/licenses/by-nc/4.0/

\section{REFERENCES}

1. Eijkemans M, Mommers M, Jos MT, et al. Physical activity and asthma: a systematic review and meta-analysis. PLOS ONE 2012;7: e50775.

2. Garcia-Aymerich J, Varraso R, Antó JM, et al. Prospective study of physical activity and risk of asthma exacerbations in older women. Am J Respir Crit Care Med 2009;179:999-1003.

3. Kilpeläinen $M$, Terho $E O$, Helenius $\mathrm{H}$, et al. Body mass index and physical activity in relation to asthma and atopic diseases in young adults. Respir Med 2006;100:1518-25

4. Strine TW, Balluz LS, Ford ES. The associations between smoking, physical inactivity, obesity, and asthma severity in the general US population. J Asthma 2007;44:651-8.

5. Teramoto M, Moonie S. Physical activity participation among adult Nevadans with self-reported asthma. J Asthma 2011;48:517-22.

6. Beckett WS, Jacobs DR Jr, Yu X, et al. Asthma is associated with weight gain in females but not males, independent of physical activity. Am J Respir Crit Care Med 2001;164:2045-50.

7. Vogelberg $\mathrm{C}$, Hirsch $\mathrm{T}$, Radon $\mathrm{K}$, et al. Leisure time activity and new onset of wheezing during adolescence. Eur Respir J 2007;30: 672-6

8. Chen $\mathrm{Y}$, Dales R, Krewski D. Leisure-time energy expenditure in asthmatics and non-asthmatics. Respir Med 2001;95:13-18.

9. Benet M, Varraso R, Kauffmann F, et al. The effects of regular physical activity on adult-onset asthma incidence in women. Respir Med 2011;105:1104-7.

10. Lucke J, Waters B, Hockey R, et al. Trends in women's risk factors and chronic conditions: findings from the Australian Longitudinal Study on Women's Health. Womens Health (Lond) 2007;3:423-32.

11. Krokstad S, Langhammer A, Hveem K, et al. Cohort profile: the HUNT study, Norway. Int J Epidemiol 2013;42:968-77.

12. Holmen J, Midthjell K, Krüger $\varnothing$, et al. The Nord-Trøndelag Health Study 1995-97 (HUNT 2): objectives, contents, methods and participation. Norsk Epidemiol 2003;13:19-32

13. Holmen J, Midthjell K, Forsen L, et al. [A health survey in Nord-Trondelag 1984-86. Participation and comparison of attendants and non-attendants]. Tidsskr Nor Laegeforen 1990;110:1973-7.

14. Driessen LM, Kiefte-de Jong JC, Jaddoe VW, et al. Physical activity and respiratory symptoms in children: the generation $\mathrm{R}$ study. Pediatr Pulmonol 2014;49:36-42.

15. Guldberg-Møller J, Hancox B, Mikkelsen D, et al. Physical fitness and amount of asthma and asthma-like symptoms from childhood to adulthood. Clin Respir J 2015;9:314-21.

16. Toren K, Brisman J, Jarvholm B. Asthma and asthma-like symptoms in adults assessed by questionnaires: a literature review. Chest 1993;104:600-9.

17. Kurtze N, Rangul V, Hustvedt BE, et al. Reliability and validity of self-reported physical activity in the Nord-Trøndelag Health Study (HUNT 2). Eur J Epidemiol 2007;22:379-87.

18. Kurtze N, Rangul V, Hustvedt BE, et al. Reliability and validity of self-reported physical activity in the Nord-Trøndelag Health StudyHUNT 1. Scand J Public Health 2008;36:52-61. 